\title{
Exosomes from Nef expressing monocytic cells restrict HIV-1 \\ replication in infected cells through the assembly of stress granules
}

\section{Mohammad Yunus Ansari, Hasan Imam, Nishant Kumar, Zulfazal Ahmed and Shahid Jameel $^{\text {*\# }}$}

Virology Group, International Centre for Genetic Engineering and Biotechnology, New Delhi, India 110067

Short Title: Nef exosomes and HIV-1 replication

*Corresponding Author: Dr. Shahid Jameel, Virology Group, ICGEB, Aruna Asaf Ali Marg, New Delhi 110067, India; Tel: +91-11-26741358/1361; Fax: +91-1126742316; Email: jameelshahid@gmail.com, shahid.jameel@wellcomedbt.org,

"Present Address: The Wellcome Trust/DBT India Alliance, 1110 DLF Tower B, Jasola District Centre, New Delhi, India; Tel: +91-11-41008402/41008403; 


\section{Abstract}

Exosomes are membranous vesicles secreted from almost all types of cells, carry proteins and nucleic acids and function as vehicles for intercellular communication. Cells infected with HIV-1 or expressing the viral Nef protein secrete more exosomes than uninfected cells or those not expressing this protein. We used stably transfected, Nef-expressing U937 human monocytic cells and exosomes purified from these cells to study their effects on HIV-1 infected and uninfected CD4+ T-cells. The Nef exosomes inhibited virus production from HIV-1 infected CD4+ T-cells, but caused activation induced cell death in uninfected bystander cells. Mutations in its conserved Arginine residues and in the secretion-modification-region failed to secrete Nef into exosomes. Cell lines expressing these mutant Nef proteins did not deliver it to the target CD4+ T-cells, and exosomes prepared from these mutant Nef-expressing cells also did not inhibit virus production. Nef exosomes inhibited virus production by inducing the assembly of stress granules in HIV-1 infected cells, which sequestered increased amounts of gag mRNA. This is a novel mechanism wherein we show the effects of exosomes on the assembly of stress granules and viral translational repression.

Key words: Nef, exosomes, HIV-1, Stress granules 


\section{Introduction}

2 The human immunodeficiency virus (HIV) continues to be a major public health

3 threat, with an estimated 35 million people living with the infection [1]. The

4 development of antiretroviral drugs is a major advance, which has improved the

5 duration as well as quality of life following HIV infection. Although therapeutic use

6 of these drugs can reduce the viral load in infected individuals to below detection

7 levels, they cannot clear the infection. Viral latency poses the biggest challenge to

8 HIV eradication [2]. During the latent phase, HIV remains integrated in the genome

9 of infected cells, but does not replicate actively and can therefore stay hidden from the

10 host immune system. Multiple signals, which are not fully understood, or

11 discontinuation of therapy may result in active transcription and viral replication [3-

12 5]. The mechanisms of latency include transcriptional regulation of viral replication

13 and microRNA (miRNA)-mediated posttranscriptional regulation of viral mRNA

14 translation and/or degradation [5-7].

15 Exosomes are 30-100 $\mathrm{nm}$ vesicles produced by the inward invagination of endosome

16 membranes in multivesicular bodies (MVBs), which fuse with the plasma membrane

17 to release exosomes into the extracellular space $[8,9]$. These are secreted from almost

18 all cell types and are present in many biological fluids including blood, saliva, breast

19 milk, urine and semen [10-13], and have been proposed as biomarkers for several

20 types of cancers [10, 14] kidney dysfunction [15] and liver disease [16]. Exosomes

21 contain many biologically active molecules including proteins, messenger RNAs

22 (mRNAs), miRNAs and lipids, and are suggested to be important for cell-to-cell

23 communication [17-19]. For example, alpha interferon (IFN- $\alpha)$ treated

24 nonparenchymal liver cells, which are resistant to hepatitis B virus (HBV) secrete 
25 exosomes that can transfer virus resistance to HBV permissive hepatocytes [20]. The

26 tumor suppressor protein PTEN is exported through exosomes and can perform its

27 function in target cells [21]. Exosomes produced from HIV-1 infected cells contain

28 the transactivation response (TAR) element and exposure of naïve cells to these

29 exosomes increases their susceptibility to HIV-1 infection [22]. The HIV-1 genome is

30 also secreted into exosomes from infected cells, and the region responsible for this

31 transfer was located at the 5 ' end of the $\mathrm{p} 17^{\mathrm{Gag}}$ open reading frame. The exosomal

32 secretion of miR29 in HIV-1 infection increases upon opiate abuse; this reduces the

33 expression of platelet-derived growth factor-beta in recipient cells and plays an

34 important role in HIV-associated neurological disorders [23].

35 The HIV-1 genome encodes the prototypic retroviral proteins (Gag, Pol and Env), two 36 regulatory proteins (Tat and Rev) and four accessory proteins (Nef, Vif, Vpr and

$37 \mathrm{Vpu}$ ). Of these, the Nef protein is expressed early in the viral life cycle and is secreted

38 into exosomes, which are found in the blood circulation of infected individuals [24].

39 Motifs in the Nef protein that are required for its packaging into exosomes have been

40 mapped to a stretch of four arginine residues (amino acids 17-22) and the sequence

41 VGFPV (amino acids 66-70) named as the secretion modification region (SMR) [25].

42 Earlier work from our laboratory has shown the Nef protein expressed to interact with

43 the miRNA silencing machinery in human monocytic U937 cells and to be packaged

44 into exosomes [26]. It also alters the mRNA and miRNA profile of exosomes [27].

45 While exosomes containing Nef were shown to cause the activation-induced death of 46 bystander CD4+ T cells [28], their effects on HIV-1 infected cells have not been

47 explored. We show here that Nef-containing exosomes inhibit viral replication in 48 infected CD4+ $\mathrm{T}$ cell lines through a post-transcriptional mechanism that involves 49 stress granule formation and translational suppression of viral mRNAs. This further 
50 emphasizes the dual effects of Nef in optimizing the cellular environment for viral

51 replication and persistence.

\section{Materials and Methods}

\section{Cells}

54 The J1.1 and U1 cells were obtained from NIH AIDS Reagent Bank and maintained 55 in RPMI containing 10\% fetal bovine serum (FBS). The U937/Nef-EYFP and 56 U937/EYFP stable cell lines were reported previously [26] and were maintained in 57 RPMI containing 10\% FBS and $350 \mathrm{ng} / \mathrm{ml}$ Puromycin. The HEK293T and TZM-bl 58 cells were maintained in DMEM containing 10\% FBS. The U937/Nef(4R/4A)-EYFP 59 and U937/Nef(VGFPV)-EYFP stable cell lines were generated in the laboratory. For 60 this, mutations within the nef gene were first generated in the pMSCV-Nef-EYFP 61 plasmid background [24] at Mutagenex Inc, NJ, USA. Retroviruses expressing Nef(4R/4A)-EYFP and Nef(VGFPV)-EYFP were generated by cotransfection of HEK293T cells with $2 \mu \mathrm{g}$ of the transfer plasmid, $1 \mu \mathrm{g}$ of pGag-Pol and $0.5 \mu \mathrm{g}$ of 64 pVSVg in a T25 flask using the calcium phosphate method. The culture supernatants 65 were collected after $36 \mathrm{hr}$ and used as the source of recombinant retroviruses. Human 66 monocytic U937 cells were washed with RPMI, starved for 90 min without serum and 67 then transduced with $500 \mu$ of culture supernatants per $1 \times 10^{6}$ cells. After a $4 \mathrm{hr}$ 68 adsorption step, the cells were washed and kept in complete medium for $48 \mathrm{hr}$ prior to 69 the addition of $350 \mathrm{ng} / \mathrm{ml}$ Puromycin. The cells were split every $48 \mathrm{hr}$ and those 70 surviving after 5 passages were used for the analysis. The clones were sorted for the

71 EYFP positive population using a Becton Dickinson Aria Cell Sorter in the Central 72 Facility of the National Institute of Immunology, New Delhi, India. The sorted clones 73 were cultured for 4-5 passages and checked for purity and EYFP expression using a 
74 Cyan-ADP flow cytometer (Beckman Coulter). Data was analyzed using Summit 4.3

75 software. Characterization of Nef function in the U937/Nef(4R/4A)-EYFP and

76 U937/Nef(VGFPV)-EYFP stable cell lines was done by flow cytometry for the

77 surface expression of CD4, MHC I, CD80 and CD86, with CD54 as a negative

78 control (data not shown).

\section{Antibody and plasmid constructs}

80 Murine anti-p24 monoclonal antibody (hybridoma supernatant) was obtained from

81 NIH AIDS Reagent Bank. APC or PE labeled antibodies for CD80 (13-0809), CD81

82 (17-0819), CD54 (13-0549) and MHC-1 (17-9876) were obtained from eBiosciences.

83 Anti-GFP (6556) antibody was purchased from Abcam. Antibodies for phospho-

84 eIF2 $\alpha$ (3597) and eIF2 $\alpha$ (9722) were from Cell Signaling, and those for G3BP1

85 (HPA004052) and APOBEC3G (HPA001812) were from Sigma. The G3BP1-GFP

86 expression plasmid was a kind gift from Dr. Jomon Joseph [29]. The p24 ELISA kit

87 was obtained from the NIH AIDS Reagent Bank.

\section{Transfection}

89 The HEK293T and TZM-bl cells were transfected using the JetPrime transfection

90 reagent according to the manufacturer's protocol. The cells were seeded in 12-well

91 plates and transfected with $200 \mathrm{ng}$ of the G3BP1-GFP plasmid. For transfection of

92 J1.1 cells a Nucleofection kit (Lonza) was used according to the manufacturer's 93 protocol.

$94 \quad$ Culture and analysis of $\mathbf{J 1 . 1}$ cells

95 For the coculture of J1.1 cells with U937/EYFP, U937/Nef-EYFP or Nef mutant cell

96 lines, $0.5 \times 10^{6}$ cells $/ \mathrm{ml}$ each of $\mathrm{J} 1.1$ cells and the U937 stable cell line were mixed in 5 
97 ml RPMI containing 10\% FBS. Supernatants were harvested from the mixed culture

98 at different time points and analyzed for p24 levels by Western blot or ELISA, and

99 the cells were analyzed by flow cytometry. For preparing conditioned media,

100 U937/EYFP and various U937/Nef-EYFP stable cell lines were seeded at a density of

$1010.5 \times 10^{6}$ cells $/ \mathrm{ml}$ in RPMI containing $10 \% \mathrm{FBS}$ for $72 \mathrm{hr}$. Cultures were centrifuged at

$1021,000 \mathrm{xg}$ for $10 \mathrm{~min}$ to remove cells and the cell-free media were diluted 1:1 with

103 fresh RPMI containing 10\% FBS. These were used as conditioned media to grow J1.1

104 cells.

\section{Purification and characterization of exosomes}

106 Exosomes were purified from culture supernatants by multiple rounds of 107 centrifugation as described earlier [26]. In brief, the U937 stable cell lines were

108 cultured in RPMI containing 10\% FBS from which contaminating exosomes and

109 microvesicles had been removed by centrifugation as described earlier [24]. The cells

110 were cultured at a density of $0.5 \times 10^{6}$ cells $/ \mathrm{ml}$ for 72 hours. These were removed by

111 centrifugation at $1,000 \times \mathrm{xg}$ for $10 \mathrm{~min}$, and the supernatants $(\mathrm{S} 1)$ were then centrifuged

112 at $15,000 \mathrm{xg}$ to remove cell debris and other aggregates. The clarified supernatants

113 were then centrifuged at $100,000 \mathrm{xg}$ to pellet exosomes. The exosomal pellet was

114 washed with ice cold PBS and resuspended in $100 \mu$ PBS for further analysis by flow

115 cytometry and Western blotting. To purify exosomes for live cell experiments, the

116 ultracentrifugation step was substituted by ultrafiltration. Briefly, $15 \mathrm{ml}$ of the culture

117 supernatants from EYFP or Nef-EYFP cell lines were concentrated through 100,000

118 MWCO amicon filters (Millipore) to a final volume of $200 \mu$ l. Flow cytometric

119 analysis of exosomes was carried out as described elsewhere [30]. Briefly, $10 \mu \mathrm{g}$ of

120 purified exosomes were coated on $4 \mu \mathrm{m}$ aldehyde/sulfate latex beads (Invitrogen) in 
121 PBS overnight at $4^{\circ} \mathrm{C}$ followed by blocking with $100 \mathrm{mM}$ glycine. The coated beads

122 were washed with PBS/0.5\% BSA and resuspended in $0.5 \mathrm{ml}$ of the same. From this,

$12310 \mu \mathrm{l}$ of the coated beads were used for flow cytometric analysis of exosomal protein

124 markers.

\section{Fluorescence imaging}

126 The formation of stress granules was analyzed by confocal microscopy. Cells (J1.1,

127 HeLa or HEK293T) were transfected with the G3BP1-GFP expression plasmid. After

$12824 \mathrm{hr}$, the transfected cells were treated with purified exosomes or conditioned media

129 from control or Nef-expressing U937 cells. Following $48 \mathrm{hr}$ of exposure, the cells

130 were washed with RPMI, fixed in $4 \%$ paraformaldehyde and mounted using antifade

131 containing DAPI (Invitrogen, Carlsbad, CA, USA). Images were acquired using a

132 Nikon A1/R confocal microscope at 60X magnification.

\section{Results}

\section{Exosomal Nef reduces HIV-1 replication in infected CD4+ $\mathbf{T}$ cell line}

135 To evaluate the effects of exosomes produced from Nef-expressing monocytic cells

136 on HIV-1 infected CD4+ T cells, we have used U937 cells stably expressing the Nef-

137 EYFP fusion protein (or EYFP as a control) [26], and J1.1 cells, which are Jurkat

138 cells latently infected with HIV-1. We first evaluated virus production from J1.1 cells

139 as a function of time when these cells were co-cultured with U937/Nef-EYFP or

140 U937/EYFP cells and. Equal volumes of the culture supernatants were collected at

141 each time and analyzed for HIV-1 in the culture medium by Western blotting for p24.

142 The result showed lower levels of HIV-1 from J1.1 cells that were cocultured with

143 U937/Nef-EYFP compared to U937/EYFP cells (Fig.1A). To confirm this was not 
144 due to activation of J1.1 cells by the control U937/EYFP cells and increased virus

145 production, we also cultured J1.1 cells alone. Equal volumes of the supernatants were

146 collected at each time and analyzed by Western blotting for $\mathrm{p} 24$. We detected similar

147 levels of virus in the culture supernatants of $\mathrm{J} 1.1$ cells alone or $\mathrm{J} 1.1$ cells co-cultured

148 with U937/EYFP and decreased virus production in case of U937/Nef-EYFP co-

149 culture (Fig. S1). The levels of virus secreted in the culture media were quantitatively

150 estimated using a p24 ELISA. There was around 50\% reduction in virus production

151 from J1.1 cells co-cultured with U937/Nef-EYFP compared to U937/EYFP cells (Fig.

152 1B). To learn if this effect was due to physical contacts between the U937 and J1.1

153 cells or due to secretory factor(s), we used cell-free conditioned media from

154 U937/Nef-EYFP or U937/EYFP cells to culture J1.1 cells. There was reduced

155 intracellular viral replication and secretion in J1.1 cells treated with conditioned

156 media from U937/Nef-EYFP compared to U937/EYFP cells (Fig. 1C and Fig. S1),

157 suggesting that secretory factor(s) might mediate this effect. Conditioned media from

158 U937/Nef-EYFP cells had no significant effects on the viability (Fig. S2A) or

159 proliferation (Fig. S2B) of J1.1 cells. Similar results were also obtained when U1

160 cells, which are U937 cells that are latently infected with HIV-1, were either co-

161 cultured or treated with conditioned media from U937/Nef-EYFP cells (Fig. S3).

162 The effects of conditioned media suggested that secretory factor(s) from Nef-

163 expressing cells mediate the down modulation of HIV-1 production from J1.1 cells.

164 Since Nef is also secreted in exosomes [27], it is plausible that these effects are

165 mediated through exosomes. We therefore isolated exosomes from the culture

166 supernatants of U937/Nef-EYFP and U937/EYFP cells (Fig. S4A), and characterized

167 the purified exosomes for surface markers with flow cytometry (Fig. S4B). Secretion

168 of the Nef protein in exosomes was confirmed by Western blotting (Fig. 1D). 
169 Exosomes produced from Nef-expressing $\mathrm{CD}^{+} \mathrm{T}$ cells were shown earlier to cause 170 apoptosis in bystander $\mathrm{CD}^{+} \mathrm{T}$ cells [28]. To functionally characterize our exosome

171 preparation, we isolated peripheral blood lymphocytes (PBLs) from donor blood,

172 treated these with exosomes from U937/Nef-EYFP or U937/EYFP cells and checked

173 for apoptosis by flow cytometry for Annexin V staining (Fig. S4C) and

174 immunoblotting for PARP cleavage (Fig. S4D). The results showed that exosomes

175 secreted from U937/Nef-EYFP cells induced apoptosis in bystander CD4+ T cells.

176 We also tested if these exosomes induced apoptosis in HIV-1 infected J1.1 cells by

177 analyzing the cleavage of procaspase 3 to caspase 3 . Surprisingly, no induction of

178 apoptosis was observed in HIV-1 infected cells treated with conditioned media from

179 U937/Nef-EYFP cells (Fig. S5A). The interaction of Nef with CXCR4 is also

180 reported to be important for inducing apoptosis in bystander CD4+ T cells [31]. We

181 therefore quantified CXCR4 levels in J1.1 cells and found no difference compared to

182 the parent Jurkat cell line (Fig. S5B).

183 To determine if Nef exosomes or some other secretory component(s) are responsible

184 for reduced viral replication in J1.1 cells, we treated J1.1 cells with exosomes or the

185 exosome-free (EF) fraction from U937/Nef-EYFP and U937/EYFP culture

186 supernatants. The result showed decreased virus production from J1.1 cells only when

187 these were treated with Nef exosomes but not with the exosome-free fraction (Fig.

188 1E). These results confirm that exosomes produced from Nef-expressing cells inhibit

189 virus production in infected CD4+ T cells.

190 The exosomal delivery of Nef to HIV-1 infected CD4+ T cells is important for

191 inhibiting viral replication 
192 Since Nef is secreted in exosomes, we asked whether the effects on viral replication

193 are linked to the presence of Nef in exosomes. To determine the role of Nef, we 194 generated U937 based cell lines that stably expressed two Nef mutants that are 195 compromised for secretion into exosomes [25]. These include the Nef-4R/4A mutant

196 with arginine to alanine changes at positions 17, 19, 21 and 22, and the Nef-VGFPV 197 mutant in which residues in the SMR region (amino acids 66-70) were changed to 198 alanine. Flow cytometry (data not shown) and Western blotting were used to confirm 199 the expression and exosomal exclusion of these mutant Nef-EYFP fusion proteins 200 (Fig. 2A).

201 Flow cytometry was then used to analyze the delivery of wild type and mutant Nef 202 proteins into J1.1 cells via exosomes. The J1.1 cells were cultured with U937 cells 203 stably expressing Nef-EYFP, Nef4R/4A-EYFP, Nef-VGFPV-EYFP or just EYFP. 204 After fixing, the mixed cell population was gated for the U937 and J1.1 cell 205 populations (Fig. S6), and the latter quantified for the EYFP signal. The results 206 showed about 30-40\% of J1.1 cells co-cultured with U937/EYFP or U937/Nef-EYFP 207 cells to be positive for the EYFP signal, but this reduced about 8- to 10-fold for J1.1 208 cells co-cultured with either U937/Nef4R/4A-EYFP or U937/Nef-VGFPV-EYFP 209 cells (Fig. 2B). These results directly indicate that Nef is efficiently transferred from 210 U937 cells to J1.1 cells through exosomes. We then looked at the effects of mutant 211 Nef proteins on down modulation of viral replication in J1.1 cells. Both Nef mutants 212 that were compromised for secretion into exosomes and transfer into J1.1 cells also 213 did not show reduced HIV-1 production from J1.1 cells, either on co-culture with 214 U937 stable cell lines or when conditioned media from U937 stable cell lines were 215 used to treat J1.1 cells (Fig. 2C). 
216 Nef exosomes induce markers of translational repression and assembly of stress

217 granules in target cells

218 To understand the mechanism(s) underlying the effects of Nef exosomes on HIV-1

219 replication, we first tested if these affect HIV-1 LTR activity. For this, J1.1 cells were

220 transfected with a HIV-1 LTR-luciferase reporter construct, and these were either co-

221 cultured or treated with conditioned media from U937/Nef-EYFP or U937/EYFP

222 cells. After $48 \mathrm{hr}$, the cells were analyzed for HIV-1 LTR driven luciferase activity.

223 No significant differences in luciferase activities were observed in either condition

224 between Nef and control exosomes (Fig. S7A and S7B). We also quantified gag

225 mRNA levels in J1.1 cells upon treatment with conditioned media or exosomes from

226 U937/Nef-EYFP or U937/EYFP cells and found no significant differences (Fig. S7C

227 and S7D). These observations suggested post-transcriptional regulation of HIV-1

228 replication by Nef-containing exosomes. To check the role of miRNAs in

229 posttranscriptional regulation of gag mRNA we examined miRNA-mediated silencing

230 of HIV-1 mRNA. For this, J1.1 cells were transfected with plasmid pMIR-Report-

231 Nef3'UTR [32], which carries the HIV-1 nef-3'UTR downstream of the luciferase

232 reporter gene; the plasmid pMIR-Report that lacked the nef-3'UTR was used as a

233 control. The transfected cells were treated with exosomes purified from U937 cells

234 that stably expressed either wild type or mutant Nef-EYFP fusion proteins or EYFP.

235 There were no significant differences in luciferase activities in J1.1 cells treated with

236 any of the exosomes (Fig. S7E).

237 We then explored the possibility of translational suppression of viral mRNAs. For

238 this, we first checked phosphorylation of the eukaryotic translation initiation factor

239 alpha (eIF2 $\alpha$ ), which is an indicator of translation arrest [33]. There was increased 
240 phosphorylation of eIF2 $\alpha$ in J1.1 cells following treatment with exosomes containing

241 Nef compared to control exosomes (Fig. 3A). It is reported that phosphorylation of

242 eIF2 $\alpha$ may lead to the assembly of stress granules, which are sites for the translational

243 repression of selected mRNAs [34]. Under such conditions the expression of G3BP1,

244 a marker of stress granules, increases and helps in their assembly. The J1.1 cells

245 treated with Nef exosomes also showed increased levels of G3BP1 compared to cells

246 treated with control exosomes (Fig 3A). Stress granules sequester selective

247 populations of mRNAs leaving out those mRNAs whose products are needed to

248 overcome stress. We did not observe any reduction in the levels of Hsp70, a

249 molecular chaperone that is needed to resolve cellular stress (Fig 3A).

250 Furthermore, we tested the assembly of stress granules by first transfecting a G3BP1-

251 GFP reporter construct in J1.1 cells followed by treatment with exosomes from Nef-

252 EYFP, mutant Nef-EYFP and EYFP cell lines. Stress granule assembly was observed

253 in J1.1 cells treated with exosomes from U937/Nef-EYFP cells, but not from control

254 cells or those that express mutant Nef proteins (Fig. 3B and Fig. S8). Similar results

255 were obtained in HeLa cells transfected with the G3BP1-GFP reporter followed by

256 treatment with different exosome populations (Fig 3B). To ascertain if gag mRNA is

257 increasingly associated with stress granules resulting in attenuated translation, we

258 immunoprecipitated stress granule and quantified the levels of gag mRNA in the

259 precipitates. Stress granules were immunoprecipitated with anti-G3BP1 antibodies,

260 total RNA associated with stress granules was isolated and gag RNA was quantified

261 by qRT-PCR. Higher levels of gag RNA were found to be associated with stress

262 granules in J1.1 cells treated with Nef exosomes compared to control exosomes (Fig.

$2633 \mathrm{C})$. 
264 The restriction factor APOBEC3G (A3G) is shown to bind HIV-1 mRNA and

265 sequester it to stress granules [35]. We also found the levels of A3G to be upregulated

266 in J1.1 cells treated with Nef exosomes (Fig. 4A), suggesting that increased levels of

267 A3G might be responsible for sequestering viral mRNA into stress granules. Since

268 A3G is also packaged in virus particles and reduces HIV-1 replication in the next

269 round of infection, we checked if increased levels of A3G have any effect on the

270 replicative ability of virions produced from J1.1 cells following their exposure to Nef

271 exosomes. For this, we took equal amounts of p24 released from J1.1 cells treated

272 with Nef or control exosomes, and checked their infectivity on TZMbl cells. Virions

273 released from $\mathrm{J} 1.1$ cells treated with Nef exosomes were about three-fold less

274 infectious than those released from cells treated with control exosomes (Fig. 4B).

\section{Discussion}

276 Several reports have established the secretion of Nef into exosomes from HIV-1

277 infected T cells, monocytes and dendritic cells as well as from cells expressing Nef

278 alone $[25,27,28]$. Nef alters membrane trafficking, increases the proliferation of

279 MVBs and enhances its own secretion in exosomes [36-38]. An earlier study has

280 shown that Nef secreted in exosomes causes activation-induced cell-death in

281 bystander (uninfected) CD4+ T cells [28]. However, the effect of Nef exosomes on

282 infected cells has not been studied. Here we analyzed the effects of Nef exosomes on

283 the HIV-1 infected J1.1 CD4+ T cell line. Our results show that exosomal Nef

284 reduces viral replication in infected cells, and that these effects are linked to the

285 secretion of Nef into exosomes and its transfer to J1.1 cells. We further show that

286 exosomal Nef does not affect proviral transcription or miRNA-mediated post-

287 transcriptional silencing of viral mRNA, but results in increased assembly of stress 
288 granules in recipient cells, implying that Nef exosomes attenuate the translation of 289 viral mRNAs in infected cells. We found decreased production of the viral Gag 290 proteins (p55 and p24) from infected J1.1 cells upon co-culture with Nef-U937 cells

291 or treatment with culture supernatant. The factor in culture supernatants responsible

292 for reduced virus replication in infected cells was found to be exosomes. These 293 nanovesicles have been reported to play important roles in intercellular 294 communication by transporting proteins, mRNAs and miRNAs to recipient cells [39].

295 Exosomes produced from HIV-1 infected cells carry the entire viral genome, 296 implicating their role in the spread of infection [40].

297 Stress granules are cytoplasmic bodies assembled in eukaryotic cells under different 298 environmental stress conditions including oxidative stress, hypoxia and infection. The 299 formation of stress granules leads to the translational arrest of select mRNAs and to 300 enhance the translation of mRNAs that are involved in relieving stress [33]. Many 301 reports have shown high levels of oxidative stress in patients with HIV-1 infection 302 [41-44]. However, the cause of systemic oxidative stress in HIV-1 infected 303 individuals has not been identified. We show that Nef exosomes induce the 304 phosphorylation of eIF2 $\alpha$ in J1.1 cells, which is a key signal for the assembly of stress 305 granules that are sites of translational suppression of selected mRNAs [45]. Exosomal 306 Nef attenuated virus production from infected cells not due to effects on HIV-1 307 transcription or miRNA-mediated translational repression, but due to the increased 308 assembly of stress granules. In support of this, RNA immunopreicpitation showed 309 increased association of gag mRNA with stress granules in infected cells that were 310 exposed to Nef exosomes. The anti-HIV restriction factor, APOBEC3G selectively 311 binds and shuttles HIV-1 mRNA between stress granules and polysomes [35]. We 
312 found increased levels of A3G in $\mathrm{J} 1.1$ cells treated with Nef exosomes and virions

313 produced from these cells showed reduced infectivity.

\section{Conclusion}

315 This study describes a novel function for Nef that is secreted in exosomes. The

316 limitation of our data is that we cannot distinguish whether this is a direct effect of

317 Nef or some other factor that is co-transported into exosomes with Nef. In either case,

318 instead of the expected Nef-mediated activation of infected cells and increased viral

319 replication, we found exosomal Nef to attenuate viral replication. This might be a

320 viral strategy to limit the activation-induced death of infected cells, and thus aid in

321 viral persistence.

\section{Author's contribution}

323 MYA performed most of the experiments, with help from HI and ZA. NK generated

324 the U937 stable cell lines expressing mutant Nef proteins. MYA and SJ analyzed and

325 interpreted the data, and wrote the manuscript.

\section{Conflict of interest and funding}

327 The authors declare that they have no competing interests. This work was supported

328 by a grant from the Department of Biotechnology, Government of India to SJ. NK

329 received a research fellowship from the Council for Scientific and Industrial

330 Research, India.

\section{Acknowledgments}

332 We thank Dr. Jomon Joseph at National Centre for Cell Sciences, Pune, India for the 333 G3BP1-GFP plasmid. 
334

335

336

337

338

339

340

341

342

343

344

345

346

347

348

349

350

3518 .

352 biology and emerging therapeutic opportunities. Nature reviews Drug discovery.

$353 \quad 2013 ; 12(5): 347-57$

354 9. G. Raposo and W. Stoorvogel. Extracellular vesicles: exosomes,

355 microvesicles, and friends. The Journal of cell biology. 2013;200(4):373-83.

356 10. C. S. Hong, L. Muller, T. L. Whiteside and M. Boyiadzis. Plasma exosomes as 357 markers of therapeutic response in patients with acute myeloid leukemia. Frontiers in 358 immunology. 2014;5:160. 
359 11. M. Salih, R. Zietse and E. J. Hoorn. Urinary Extracellular Vesicles and the

360 Kidney: Biomarkers and Beyond. American journal of physiology Renal physiology.

3612014.

362 12. L. Vojtech, S. Woo, S. Hughes, et al. Exosomes in human semen carry a

363 distinctive repertoire of small non-coding RNAs with potential regulatory functions.

$364 \quad$ Nucleic acids research. 2014.

365 13. C. Lasser, V. S. Alikhani, K. Ekstrom, et al. Human saliva, plasma and breast

366 milk exosomes contain RNA: uptake by macrophages. Journal of translational

367 medicine. 2011;9:9.

368 14. D. Duijvesz, K. E. Burnum-Johnson, M. A. Gritsenko, et al. Proteomic

369 profiling of exosomes leads to the identification of novel biomarkers for prostate

$370 \quad$ cancer. PloS one. 2013;8(12):e82589.

371 15. S. Alvarez, C. Suazo, A. Boltansky, et al. Urinary exosomes as a source of

372 kidney dysfunction biomarker in renal transplantation. Transplantation proceedings.

$373 \quad 2013 ; 45(10): 3719-23$.

374 16. E. Rodriguez-Suarez, E. Gonzalez, C. Hughes, et al. Quantitative proteomic

375 analysis of hepatocyte-secreted extracellular vesicles reveals candidate markers for

376 liver toxicity. Journal of proteomics. 2014;103C:227-40.

377 17. N. Kosaka, Y. Yoshioka, K. Hagiwara, et al. Trash or Treasure: extracellular

378 microRNAs and cell-to-cell communication. Frontiers in genetics. 2013;4:173.

379 18. K. J. Rayner and E. J. Hennessy. Extracellular communication via microRNA:

380 lipid particles have a new message. Journal of lipid research. 2013;54(5):1174-81.

381 19. G. Turturici, R. Tinnirello, G. Sconzo and F. Geraci. Extracellular membrane

382 vesicles as a mechanism of cell-to-cell communication: advantages and 
383 disadvantages. American journal of physiology Cell physiology. 2014;306(7):C621-

38433.

385 20. J. Li, K. Liu, Y. Liu, et al. Exosomes mediate the cell-to-cell transmission of

386 IFN-alpha-induced antiviral activity. Nature immunology. 2013;14(8):793-803.

387 21. U. Putz, J. Howitt, A. Doan, et al. The tumor suppressor PTEN is exported in

388 exosomes and has phosphatase activity in recipient cells. Science signaling.

$389 \quad 2012 ; 5(243):$ ra70.

390 22. A. Narayanan, S. Iordanskiy, R. Das, et al. Exosomes derived from HIV-1-

391 infected cells contain trans-activation response element RNA. The Journal of

392 biological chemistry. 2013;288(27):20014-33.

393 23. G. Hu, H. Yao, A. D. Chaudhuri, et al. Exosome-mediated shuttling of

394 microRNA-29 regulates HIV Tat and morphine-mediated neuronal dysfunction. Cell

395 death \& disease. 2012;3:e381.

396 24. Y. Fujii, K. Otake, M. Tashiro and A. Adachi. Soluble Nef antigen of HIV-1 is

397 cytotoxic for human CD4+ T cells. FEBS letters. 1996;393(1):93-6.

398 25. S. A. Ali, M. B. Huang, P. E. Campbell, et al. Genetic characterization of HIV

399 type 1 Nef-induced vesicle secretion. AIDS research and human retroviruses.

$400 \quad 2010 ; 26(2): 173-92$.

401 26. M. Aqil, A. R. Naqvi, A. S. Bano and S. Jameel. The HIV-1 Nef protein binds

402 argonaute-2 and functions as a viral suppressor of RNA interference. PloS one.

$403 \quad 2013 ; 8(9):$ e74472.

404 27. M. Aqil, A. R. Naqvi, S. Mallik, et al. The HIV Nef protein modulates cellular

405 and exosomal miRNA profiles in human monocytic cells. Journal of extracellular

406 vesicles. 2014;3. 
407 28. M. Lenassi, G. Cagney, M. Liao, et al. HIV Nef is secreted in exosomes and

408 triggers apoptosis in bystander CD4+ T cells. Traffic. 2010;11(1):110-22.

409 29. P. K. Sahoo, P. Murawala, P. T. Sawale, et al. Wnt signalling antagonizes

410 stress granule assembly through a Dishevelled-dependent mechanism. Biology open.

$411 \quad 2012 ; 1(2): 109-19$.

412 30. C. Thery, S. Amigorena, G. Raposo and A. Clayton. Isolation and

413 characterization of exosomes from cell culture supernatants and biological fluids.

414 Current protocols in cell biology / editorial board, Juan S Bonifacino [et al].

415 2006; Chapter 3:Unit 322.

416 31. C. O. James, M. B. Huang, M. Khan, et al. Extracellular Nef protein targets

417 CD4+ T cells for apoptosis by interacting with CXCR4 surface receptors. Journal of

418 virology. 2004;78(6):3099-109.

419 32. J. K. Ahluwalia, S. Z. Khan, K. Soni, et al. Human cellular microRNA hsa-

420 miR-29a interferes with viral nef protein expression and HIV-1 replication.

421 Retrovirology. 2008;5:117.

422 33. P. Anderson and N. Kedersha. Stress granules. Current biology : CB.

$423 \quad 2009 ; 19(10):$ R397-8.

424 34. F. Valiente-Echeverria, L. Melnychuk and A. J. Mouland. Viral modulation of 425 stress granules. Virus research. 2012;169(2):430-7.

426 35. S. L. Kozak, M. Marin, K. M. Rose, C. Bystrom and D. Kabat. The anti-HIV-

4271 editing enzyme APOBEC3G binds HIV-1 RNA and messenger RNAs that shuttle

428 between polysomes and stress granules. The Journal of biological chemistry.

429 2006;281(39):29105-19. 
430 36. J. F. Roeth and K. L. Collins. Human immunodeficiency virus type 1 Nef:

431 adapting to intracellular trafficking pathways. Microbiology and molecular biology

432 reviews : MMBR. 2006;70(2):548-63.

433 37. P. Stumptner-Cuvelette, M. Jouve, J. Helft, et al. Human immunodeficiency

434 virus-1 Nef expression induces intracellular accumulation of multivesicular bodies

435 and major histocompatibility complex class II complexes: potential role of

436 phosphatidylinositol 3-kinase. Molecular biology of the cell. 2003;14(12):4857-70.

437 38. L. J. Costa, N. Chen, A. Lopes, et al. Interactions between Nef and AIP1

438 proliferate multivesicular bodies and facilitate egress of HIV-1. Retrovirology.

$4392006 ; 3: 33$.

440 39. C. Bang and T. Thum. Exosomes: new players in cell-cell communication.

441 The international journal of biochemistry \& cell biology. 2012;44(11):2060-4.

442 40. S. Columba Cabezas and M. Federico. Sequences within RNA coding for

443 HIV-1 Gag p17 are efficiently targeted to exosomes. Cellular microbiology.

$444 \quad 2013 ; 15(3): 412-29$.

445 41. D. Morris, J. Ly, P. T. Chi, et al. Glutathione synthesis is compromised in

446 erythrocytes from individuals with HIV. Frontiers in pharmacology. 2014;5:73.

447 42. A. M. Tang and E. Smit. Oxidative stress in HIV-1-infected injection drug

448 users. Journal of acquired immune deficiency syndromes. 2000;25 Suppl 1:S12-8.

449 43. W. E. Stehbens. Oxidative stress in viral hepatitis and AIDS. Experimental

450 and molecular pathology. 2004;77(2):121-32.

451 44. G. W. Pace and C. D. Leaf. The role of oxidative stress in HIV disease. Free

452 radical biology \& medicine. 1995;19(4):523-8. 
453 45. N. L. Kedersha, M. Gupta, W. Li, I. Miller and P. Anderson. RNA-binding

454 proteins TIA-1 and TIAR link the phosphorylation of eIF-2 alpha to the assembly of

455 mammalian stress granules. The Journal of cell biology. 1999;147(7):1431-42.

456

457 Figure Legends

458

459 Figure 1: Exosomes from Nef-expressing cells inhibit virus production from

460 infected cells. (A) J1.1 cells were co-cultured with U937/EYFP cells (lanes J1.1-

461 YFP) or U937/Nef-EYFP cells (lanes J1.1-Nef). The culture supernatants were

462 collected at different times and analyzed by Western blotting for p24. (B) The

463 coculture supernatants from J1.1 and either U937/Nef-EYFP cells (J1.1-Nef) or

464 U937/EYFP cells (lanes J1.1-YFP) were quantified with ELISA for p24 levels.

465 Results are shown as mean of three independent experiments, each with triplicate

466 measurements $(* \mathrm{p}<0.05)$. (C) J1.1 cells were cultured in conditioned media from

467 U937/EYFP cells (lanes J1.1-YFP) or U937/Nef-EYFP cells (lanes J1.1-Nef) for 72

468 hr. Cells and supernatants were harvested and analyzed for intracellular p24/p55 and

469 p24, respectively. Beta-actin served as a loading control for cell lysates. (D)

470 Exosomes were purified from U937/EYFP or U937/Nef-EYFP cells and Western

471 blotting was carried out with anti-GFP antibodies; the EYFP and Nef-EYFP are

472 indicated. (E) J1.1 cells were treated with exosome-free media or exosomes from

473 U937/EYFP cells (lanes J1.1-YFP) or U937/Nef-EYFP cells (lanes J1.1-Nef) for 72

$474 \mathrm{hr}$, and p24 in the culture supernatants was analysed by Western blotting.

475 Figure 2: Exosomal secretion of the Nef protein and its delivery into target cells.

476 (a) U937 cell lines that stably expressed the two Nef mutants (4R/4A and VGFPV)

477 were generated. The expression of wild type and mutant Nef proteins in the cell 
478 lysates and exosomes purified from these cell lines were evaluated by Western

479 blotting with anti-GFP antibodies; the EYFP and Nef-EYFP are indicated. Beta-actin

480 served as a loading control. Exosomes were also marked by Western blotting for Alix.

481 (B) Delivery of the Nef protein through exosomes was analyzed by coculturing J1.1

482 cells with U937 cells stably expressing EYFP, Nef and the two Nef mutants followed

483 by flow cytometry for EYFP in J1.1 cells. The J1.1 and U937 cell populations were

484 separated based on forward scatter as shown in Supplementary Figure 6. The numbers

485 indicate percent J1.1 cells positive for EYFP. (C) J1.1 cells were either cocultured

486 with or treated with conditioned media (CM) from U937/EYFP, U937/Nef-EYFP,

487 U937-Nef(4R/4A)-EYFP or U937-Nef(VGFPV)-EYFP cells. The culture

488 supernatants were collected after $72 \mathrm{hr}$ and analyzed for p24 by Western blotting.

489 Figure 3: Nef exosomes induce stress granules assembly and sequestration of

490 HIV-1 gag mRNA in J1.1 cells. (A) J1.1 cells were treated with conditioned media

491 from U937/EYFP cells (J1.1-YFP) or U937/Nef-EYFP cells (J1.1-Nef) for $72 \mathrm{hr}$. The

492 cell lysates were then analyzed for phosphor-eIF2 $\alpha$, total eIF2 $\alpha$, G3BP1 (stress

493 granule marker) and HSP70 levels by Western blotting. Beta-actin served as a loading

494 control. (B) J1.1 cells were transfected with the G3BP1-GFP plasmid and seeded in a

495 12-well plate, followed by treatment with exosomes purified from U937/EYFP,

496 U937/Nef-EYFP, U937-Nef(4R/4A)-EYFP or U937-Nef(VGFPV)-EYFP cells.

497 Assembly of stress granules (arrows) was checked by confocal microscopy. (C) Stress

498 granules were immunoprecipitated with anti-G3BP1 antibody and association of gag

499 mRNA with stress granules was analyzed by qRT-PCR. Left panel shows Western

500 blot of immunoprecipitation to confirm pull down of G3BP1. Input G3BP1 and actin

501 were used as control. Right panel shows the fold enrichment of gag mRNA in stress 
502 granules. RNA was isolated from anti-G3BP1 immunoprecipitate by Trizol-

503 Chloroform and analyzed for gag mRNA by qRT-PCR $(* * * \mathrm{p}<0.005)$.

504 Figure 4: J1.1 cells treated with Nef exosomes produce less infectious virus. (A)

$505 \mathrm{~J} 1.1$ cells were treated with conditioned media from U937/EYFP cells (J1.1-YFP) or

506 U937/Nef-EYFP cells (J1.1-Nef) for $72 \mathrm{hr}$. The cell lysates were then analyzed for

507 APOBEC3G levels by Western blotting. Beta-actin served as a loading control. (B)

508 Viruses in the culture media of J1.1 cells after treatment with conditioned media from

509 U937/EYFP cells (J1.1-EYFP) or U937/Nef-EYFP cells (J1.1-Nef) for $72 \mathrm{hr}$, were

510 assayed for infectivity on TZMbl cells and the values were normalized to p24 levels.

511 Results are shown as mean of three independent experiments, each with triplicate

512 measurements $(* \mathrm{p}<0.05)$. 
A.
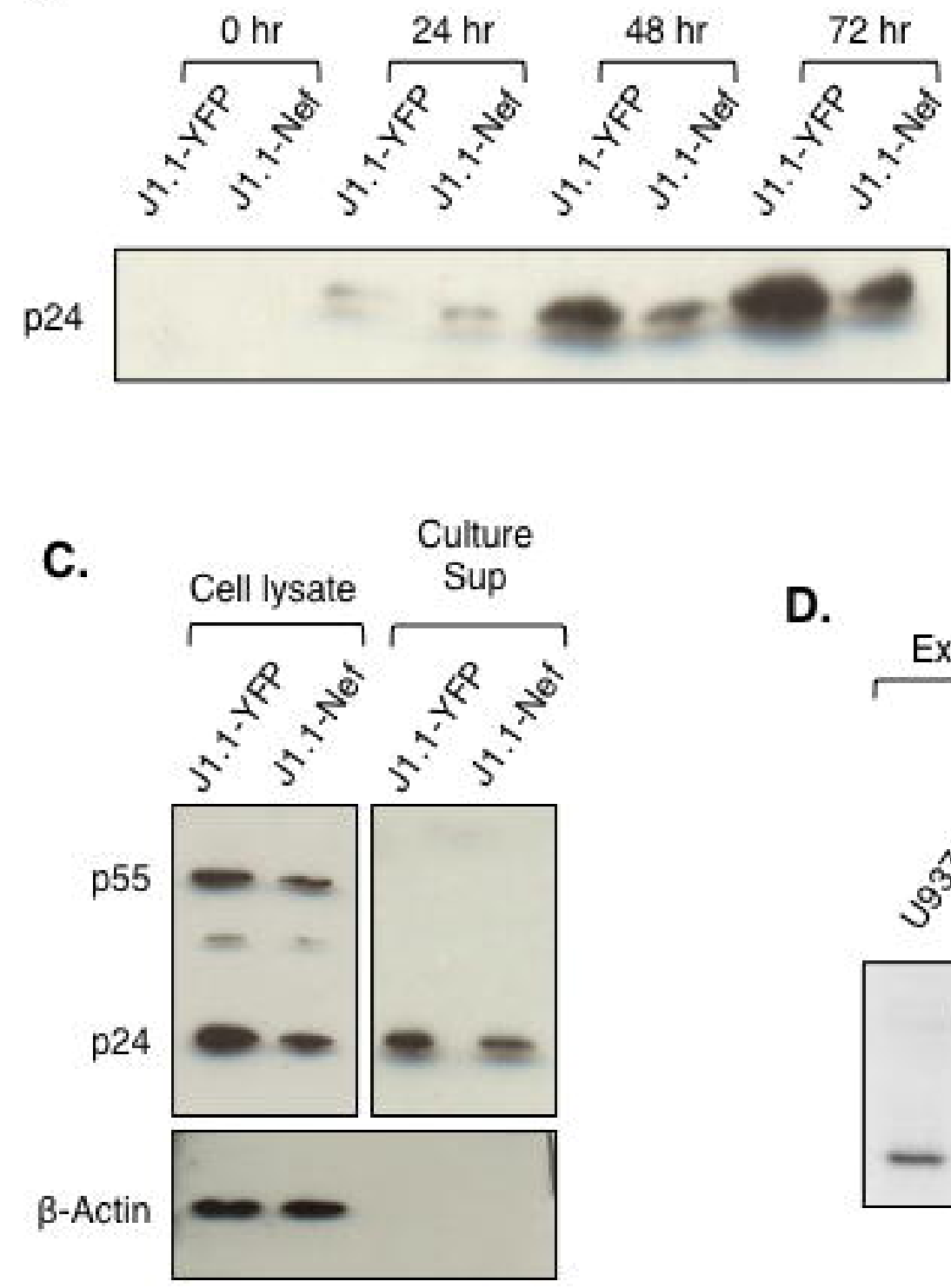

D.
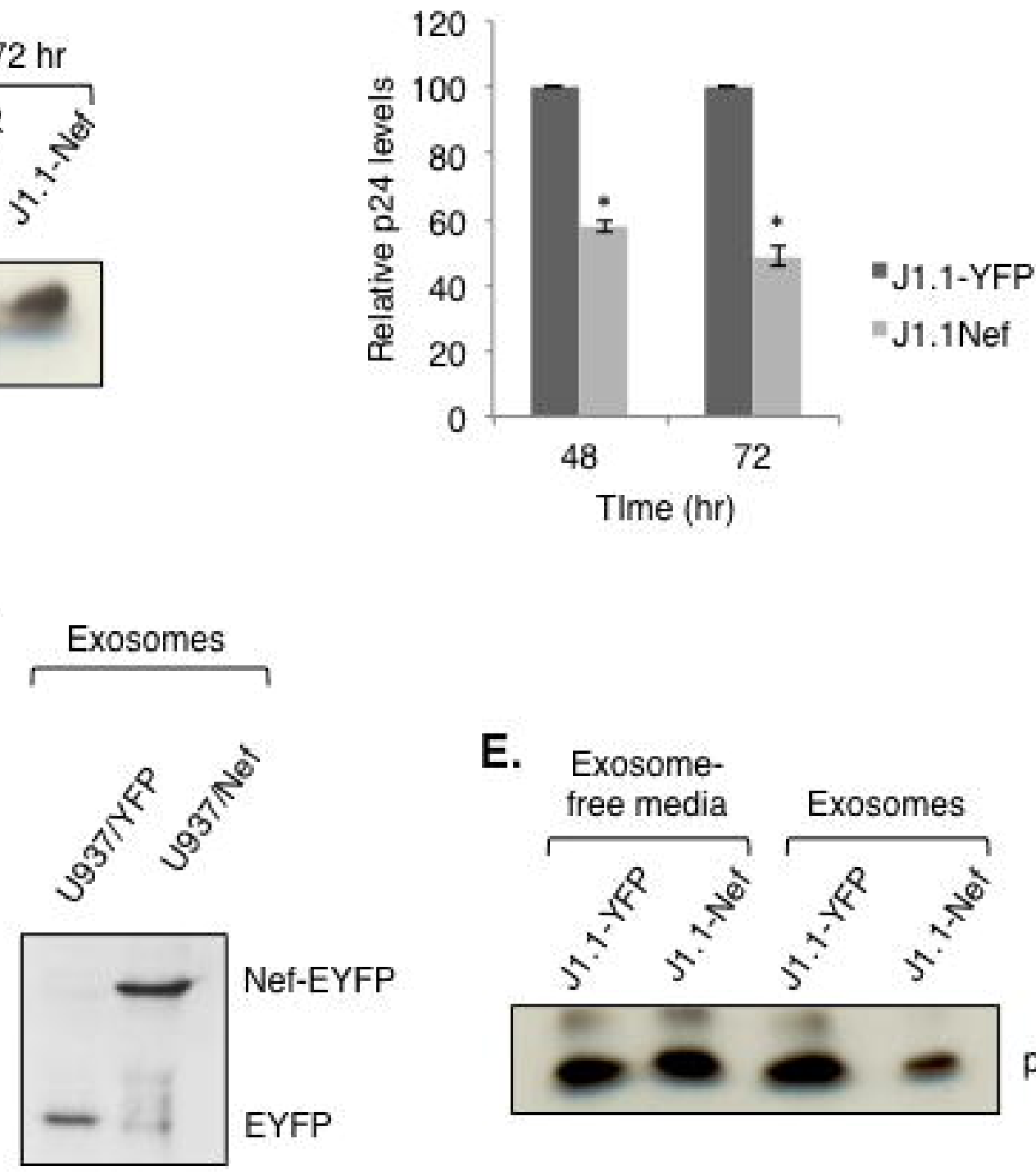
A.

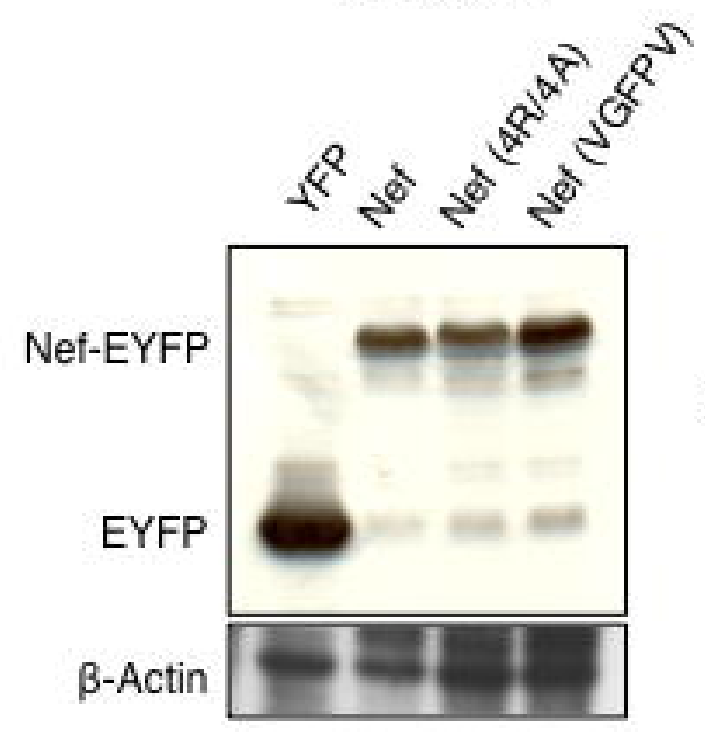

Exosomes

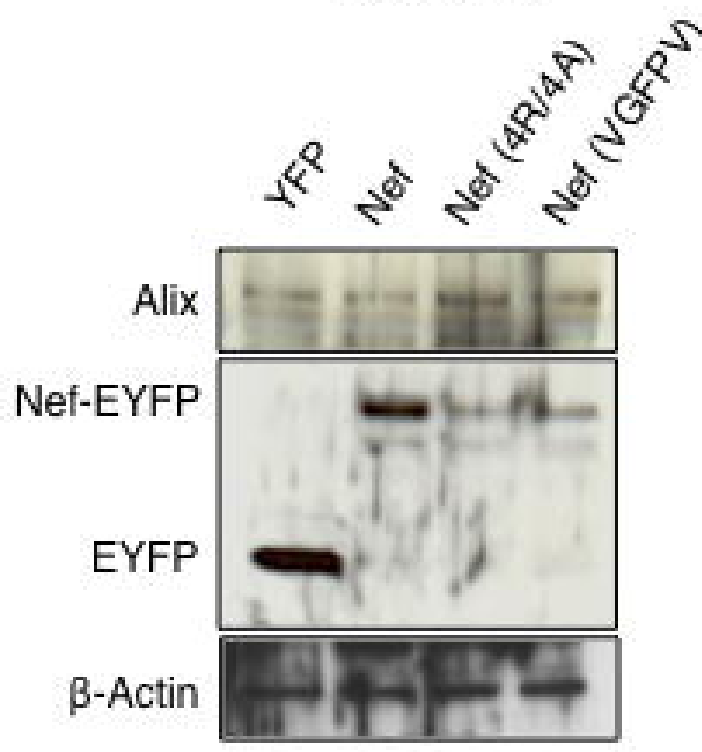

B.
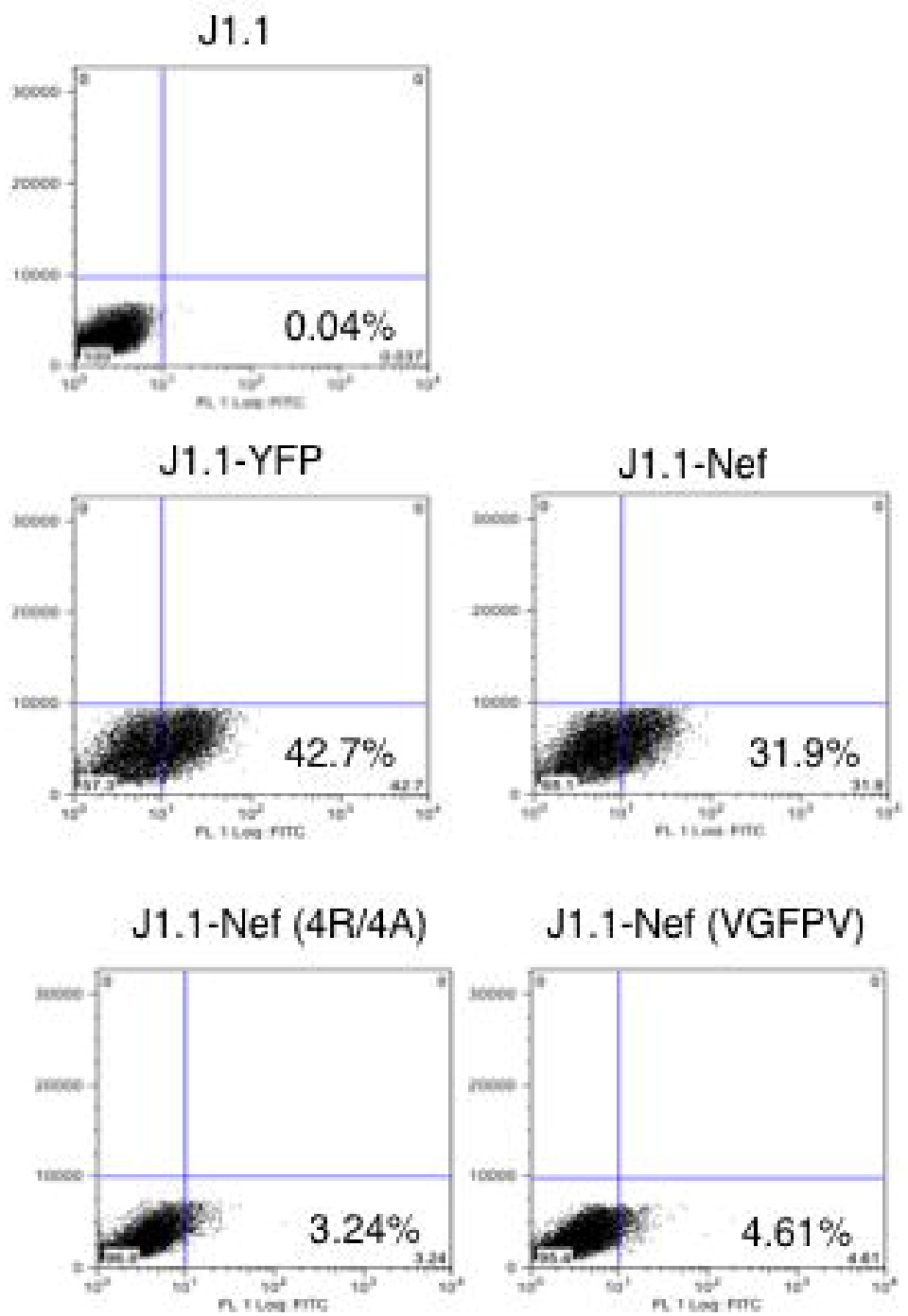

J1.1-Nef (VGFPV)

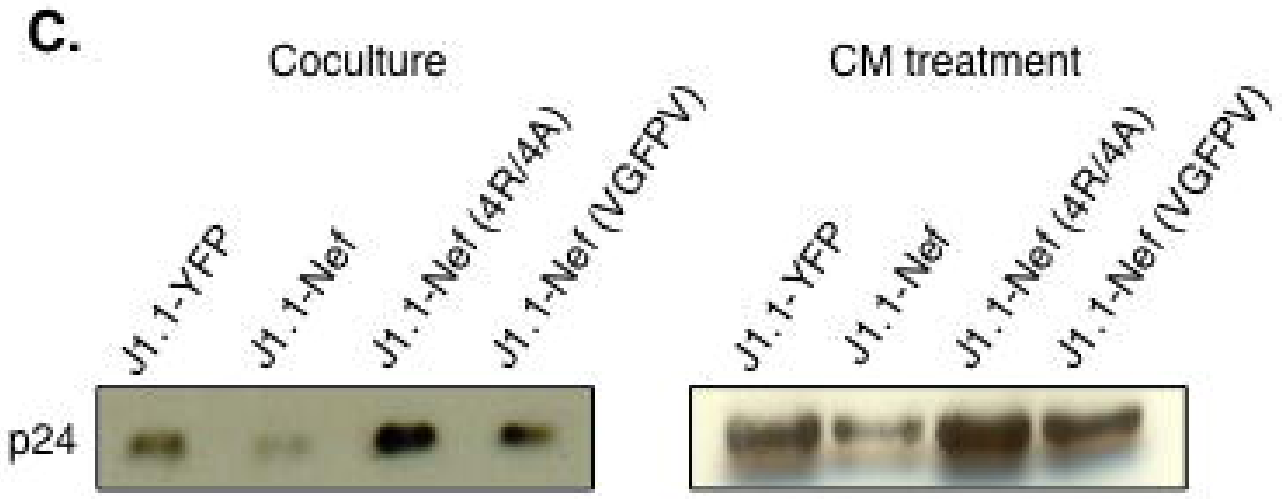




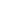




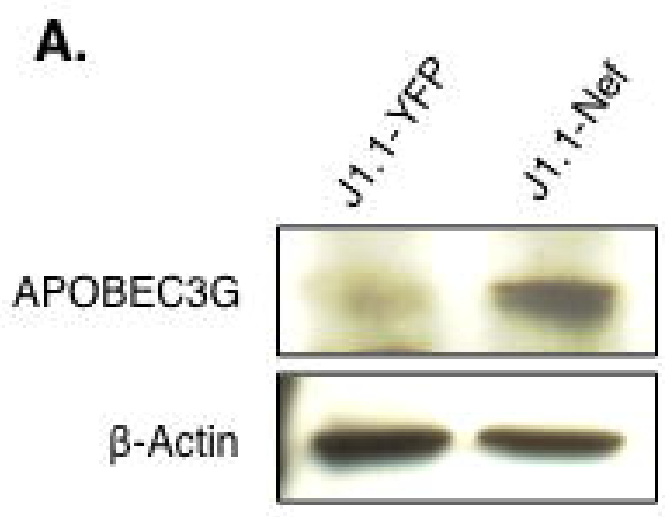

B.

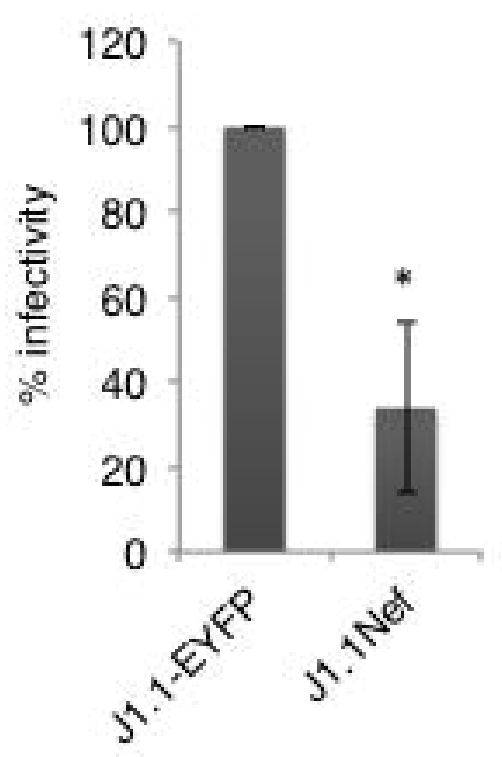

Figure 4 